\title{
A FOTOQUÍMICA BÁSICA E APLICADA NO BRASIL DURANTE OS ÚLTIMOS 40 ANOS
}

\author{
Miguel G. Neumann e Carla Cristina Schmitt* \\ Departamento de Físico-Química, Instituto de Química de São Carlos, Universidade de São Paulo, Caixa Postal 780, 13560-970 \\ São Carlos - SP, Brasil
}

Recebido em 01/04/2017; aceito em 10/05/2017

\begin{abstract}
FUNDAMENTAL AND APPLIED PHOTOCHEMISTRY DURING THE LAST 40 YEARS IN BRAZIL. This paper describes the activities in the area of Photochemistry in Brazil, from its origins at the beginning of the last Century until now. The original Research Groups (formed until 1980) in this area, as well as those created afterwards are indicated. Different orientations and specialties in photochemistry, and their extensions and uses in other fundamental and applied areas are mentioned. The number of indexed scientific papers published by photochemists based in Brazil increased from 30 to 130 during the 2000-2016 period.
\end{abstract}

Keywords: photochemistry; photobiology; photobiochemistry; chemiluminescence, photophysics

\section{INTRODUÇÃO}

A SBQ, na celebração de seus 25 anos, publicou uma edição comemorativa na Revista Química Nova apresentando o desempenho das diversas áreas da química no período e as suas perspectivas futuras. Na ocasião, Neumann e Quina ${ }^{1}$ escreveram um artigo fazendo considerações e descrevendo o histórico da área de fotoquímica. Nesse artigo foram destacados pontos importantes da origem da Fotoquímica no Brasil pelos autores. Assim, a primeira parte deste trabalho apresenta uma releitura do manuscrito "Fotoquímica no Brasil" de 2002, referente aos anos de 1971-2001. A segunda parte é dedicada a descrever os avanços da área nos últimos 15 anos.

\section{RELEITURA DA FOTOQUÍMICA NO BRASIL DE 1971-2001}

Neumann e Quina ${ }^{1}$ em seu trabalho apresentaram uma retrospectiva histórica do surgimento da fotoquímica no Brasil. Os primórdios da área começaram com a chegada dos pioneiros Dr. Hans Stamreich (1902-1962) e Dr. Hans Zocher (1893-1969) e se estenderam até o surgimento das primeiras linhas formais de fotoquímica incentivadas

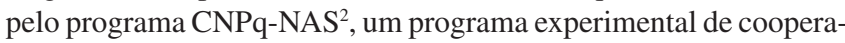
ção internacional entre o CNPq e a National Academy of Sciences dos Estados Unidos que, na época, apoiou a consolidação da fotoquímica como uma das áreas prioritárias.

Assim, por um lado, a partir da cooperação de pesquisadores do lado brasileiro (Profs. Drs. W. Bruce Kover e Vicente G. Toscano) e do lado americano (Prof. Dr. Georg S. Hammond, CALTECH) surgiram os primeiros Laboratórios de Fotoquímica Orgânica no Brasil no IQ-UFRJ e IQ-USP. Na época se agregaram a esses Laboratórios recém-doutores americanos alguns no Rio de Janeiro (David Mog e David Nicodem) e em São Paulo (Richard G. Weiss e Frank Quina) que cooperaram nos aspectos científicos e acadêmicos. Ambos laboratórios continuam atuantes e permanecem ainda hoje difundindo pesquisas nas áreas de fotoquímica.

Por outro lado, a cooperação do Programa CNPq-NAS ${ }^{2}$ incentivou a área de química de coordenação. Nesta cooperação houve a participação de pesquisadores como os Profs. Drs. Henry Taube (Stanford University), Harry B. Gray (CALTECH) e Ernesto Giesbrecht (IQ-USP). Apesar de não ser o objetivo central da

*e-mail: carla@iqsc.usp.br pesquisa deles a fotoquímica inorgânica, foi levada em conta a importância tanto da fotoquímica como da fotofísica na química de coordenação. Desse modo, pode-se inferir que grande parte dos grupos de fotoquímica inorgânica que surgiram no estado de São Paulo tiveram de alguma maneira alguma relação com o laboratório estabelecido no IQ-USP ${ }^{1}$.

O Programa CNPq-NAS, foi criado num momento propício já que coincidiu com a criação dos programas formais de pós-graduação no Brasil ${ }^{2}$, estabelecendo-se a fotoquímica como uma das áreas de maior interesse. Desse modo, no período de 1971 até 1980 foram criados novos núcleos de pesquisa dentre os quais se pode destacar o Grupo de Fotoquímica do IFQSC, USP-São Carlos que veio a ter uma contribuição significativa como agente multiplicador. Outros grupos independentes surgiram em áreas complementares da fotoquímica, podendo-se destacar: C.T. Lin (UNICAMP) trabalhando com a fotoquímica induzida por lasers, I. Brinn (UFRJ) atuando em fotofísica, M. A. De Paoli (UNICAMP) investigando fotoquímica de compostos de coordenação, G. Cilento e R. Meneghini investigando os fenômenos fotobioquímica ambos no IQ-USP e R. Tyrrell e L. Caldas dedicados à área de fotobiologia no Rio de Janeiro. Estes grupos progrediram rapidamente devido a que seus líderes que eram pesquisadores com larga experiência no exterior, contribuindo para a acelerada formação de uma massa crítica de competência em fotoquímica.

Findo esse período inicial da establecimento dos primeiros laboratórios dedicados à fotoquímica, as décadas de 80 e 90 significaram o fortalecimento dos grupos já existentes e a criaçaõ de novos grupos com destaque ao interior paulista, com na UNICAMP, USP (São Carlos e Ribeirão Preto) e UNESP (Araraquara e São José do Rio Preto) e no Rio de Janeiro a UFRRJ, em Seropédica.

Por volta dos anos 90 o espectrofluorímetro era o principal equipamento usado nas pesquisas em fotoquímica e o mais comum de ser encontrado nos laboratórios de pesquisas dedicados à área. A Figura 1 apresenta a imagem de um espectrofluorimetro da Aminco-Bowman J4-8960A utilizado nesse período.

$\mathrm{Na}$ época ainda havia alguns sistemas para determinação de rendimentos quânticos de fotorreação, um sistema analógico para a determinação de tempos de vida de fluorescência no IQ-USP e um equipamento de fotólise relâmpago convencional da Applied Photophysics (modelo KN020) com resolução em microssegundos que estava alocado no Laboratório de Fotoquímica do IFQSC/USP, Figura 2. 


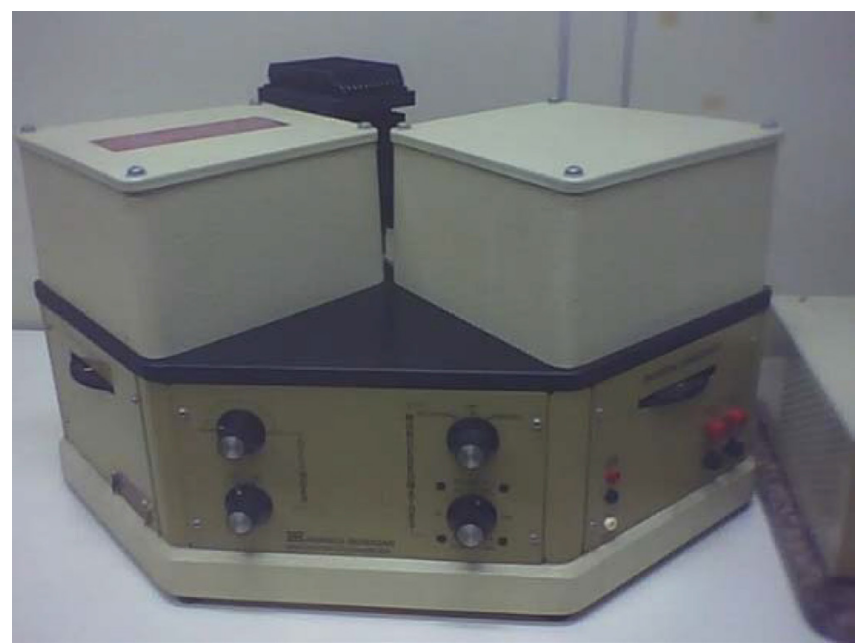

Figura 1. Imagem de um espectrofluorimetro Aminco-Bowman J4-8960A utilizado entre 1983-1992 no Laboratório de Fotoquímica do IFQSC/USP

O primeiro fórum dedicado à área de fotoquímica no Brasil acontecu em 1981 com o "Primeiro Encontro Informal Brasileiro de Fotoquímica e Fotobiologia" (EIBhv) ocorrido em Campinas que foi seguido de outros nos anos de 1983 e 1986 que aconteceram em São Paulo e em São Carlos, respectivamente. Além desses eventos de caráter nacional, foi realizado em $1982 \mathrm{em}$ Santiago do Chile o "Primeiro Encontro Latino-Americano de Fotoquímica e Fotobiologia" (ELAFOT). Este encontro reuniu fotoquímicos chilenos, argentinos e brasileiros. Este primeiro encontro estabeleceu uma integração estreita entre os grupos latinoamericanos. Dessa maneira, em 1988 ocorreu em São Carlos o Segundo ELAFOT e Quarto EIBhv. Este encontro contou com a participação de mais ou menos 50 participantes, incluindo-se entre eles vários pesquisadores estrangeiros, como Andre Braun (Suiça), Frans de Schryver (Bélgica), Tito Scaiano (Canadá), Silvia E. Braslavsky (Alemanha), Franco Scandola (Itália) e Dick Weiss (Estados Unidos). A partir deste evento as relações entre os pesquisadores brasileiros e estrangeiros foram estreitadas por meio de intercâmbios e projetos de cooperação internacional. A Figura 3 apresenta a foto oficial do evento.

A inserção da comunidade fotoquímica brasileira na América Latina vem sendo mantida ao longo das décadas por meio dos ELAFOTs, que neste ano terá sua XIII edição, destacando-se que quatro destes eventos foram realizados no Brasil. A Tabela I apresenta os anos e localização dos Encontros do ELAFOT ${ }^{3}$.

Além dos Encontros Latino-americanos, a comunidade brasileira de fotoquímica sediou dois encontros da Inter-American Photochemical Society (I-APS) em 1996 (Foz de Iguaçu) e 2006 (Salvador). Em 1996, quando da realização do I Encontro Sul Americano do I-APS, foi criado um fundo especial (G. Cilento Award Fund) para facilitar a participação de jovens cientistas que trabalham na America Latina nas conferências anuais do I-APS. Desde a sua criação a comunidade fotoquímica brasileira teve quatro jovens cientistas premiados: Pedro Camargo (2013), Carla Santos de Oliveira (2008), Sahari B. Yamaki (2004) e Ana Paola P. Cione (2000), evidenciando que a pesquisa em fotoquímica no país vem sendo atuante e incentivando novos talentos ao longo dos anos.

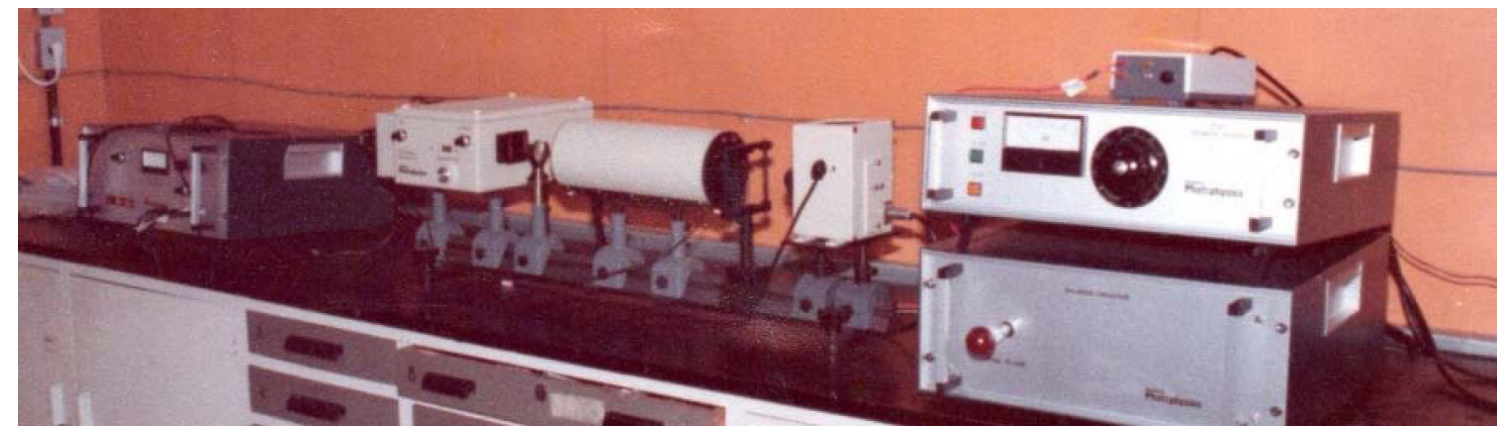

Figura 2. Imagem do equipamento de fotólise relâmpago convencional, resolução em microssegundos, utilizado entre 1983-1992 no Laboratório de Fotoquímica do IFQSC/USP

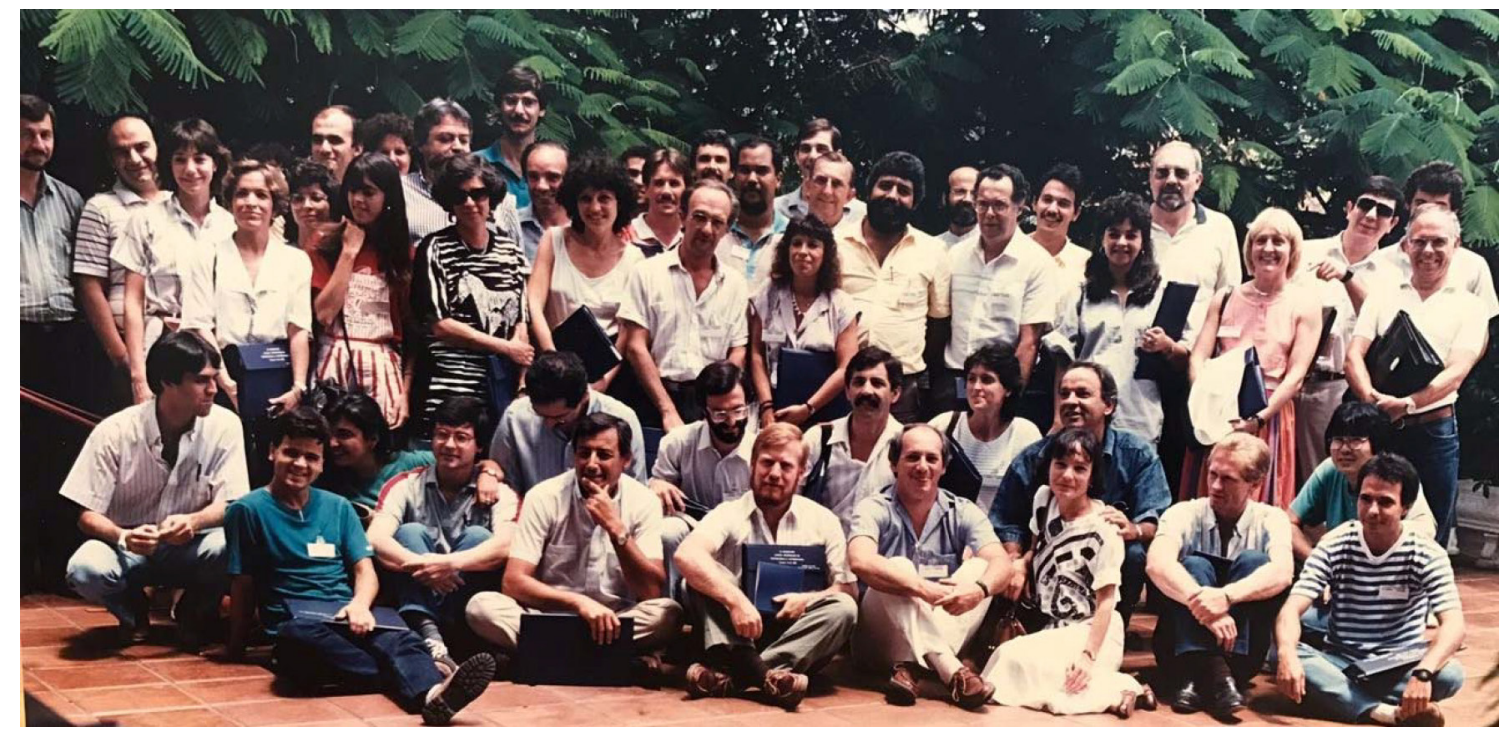

Figura 3. Fotografia oficial do Segundo ELAFOT e Quarto EIBhv realizado em São Carlos em 1988 
Tabela 1. Os anos e a localização dos Encontros do ELAFOT

\begin{tabular}{ccc}
\hline ELAFOT & LOCAL & ANO \\
\hline I & Santiago, Chile & 1982 \\
II & São Carlos, Brasil & 1988 \\
III & Mar del Plata, Argentina & 1991 \\
IV & Valparaiso, Chile & 1994 \\
V & Los Cocos, Argentina & 1997 \\
VI & Teresópolis, Brasil & 1999 \\
VII & Viña del Mar, Chile & 2002 \\
VIII & La Plata, Argentina & 2004 \\
IX & Cubatão, Brasil & 2008 \\
X & Valparaiso, Chile & 2010 \\
XI & Córdoba, Argentina & 2012 \\
XII & Maresias, Brasil & 2015 \\
XIII & Córdoba, Argentina & 2017 (a ser realizado) \\
\hline
\end{tabular}

Desde que surgiu a área de fotoquímica no Brasil, uma de suas caracteristicas marcantes tem sido o elevado grau de interdisciplinaridade. Assim, ao longo de muitos anos os eventos de fotoquímica continuam reunindo pesquisadores de diversas áreas tais como: quântica, espectroscopia, bioquímica, biofísica, ambiental, analítica, materiais, biológica entre outras. Como exemplo pode-se destacar o Encontro sobre Aplicações Ambientais de Processos Oxidativos Avançados (EPOA) que teve sua primeira edição em 2001 e encontra-se na VIII edição realizada no ano de 2015 sendo a temática os aspectos ambientais da fotoquímica.

Os anos 90 foram de crescimento próspero para a área de fotoquímica, sendo que o número de publicações de pesquisadores nas diversas especialidades de fotoquímica no Brasil em revistas internacionais saltou de 0,5 artigos por ano nas décadas anteriores para 6,5 artigos por ano após $1990^{1}$. O período foi também marcado pela criação em 1992 de uma Seção própria na área de Fotoquímica dentro da Sociedade Brasileira de Química (SBQ).

Nesse período o país experimentou crescimento e estabilidade econômica, e nesse contexto um elemento fundamental para desenvolvimento do campo de fotoquímica foi a disponibilidade de recursos para a modernização dos equipamentos essenciais para o desenvolvimento de trabalhos competitivos. Assim, equipamentos para a determinação de tempos de vida de fluorescência, sistemas de fotólise por pulso de laser (Nd-YAG) e sistemas para a detecção direta do oxigênio singlete ficaram disponíveis para serem utilizados nas pesquisas nos diversos grupos atuantes na área.

Neste período pode-se observar que a fotoquímica no Brasil alcançou seu amadurecimento demonstrado pela criação de grupos consolidados e equipamentos para estudos fotofísicos disponíveis nos principais centros do país permitindo que pesquisas na área fossem igualadas com centros de excelência internacionais. Nesse período, destaca-se ainda o crescimento do número de grupos que passaram a utilizar as metodologias de fotoquímica e fotofísica em suas pesquisas ${ }^{1}$.

No final da década de 90 e início de 2000 as áreas principais de pesquisas em fotoquímica e fotofísica estavam centradas no eixo RioSão Paulo com alguns grupos pontuais fora desse eixo ${ }^{1}$. As grandes áreas de atuação em fotoquímica no país estão apresentadas na Figura 4 bem como as instituições envolvidas com cada tema.

Nesse período, foi feita uma reflexão sobre as perspectivas da fotoquímica no país para as próximas décadas, assim como qual seria a melhor forma de acompanhar a evolução da área no cenário internacional. Assim, previa-se que estudos fotoquímicos e fotofísicos passariam a se tornar uma técnica avançada para solucionar problemas de outros campos da ciência. Esperava-se que os estudos clássicos de
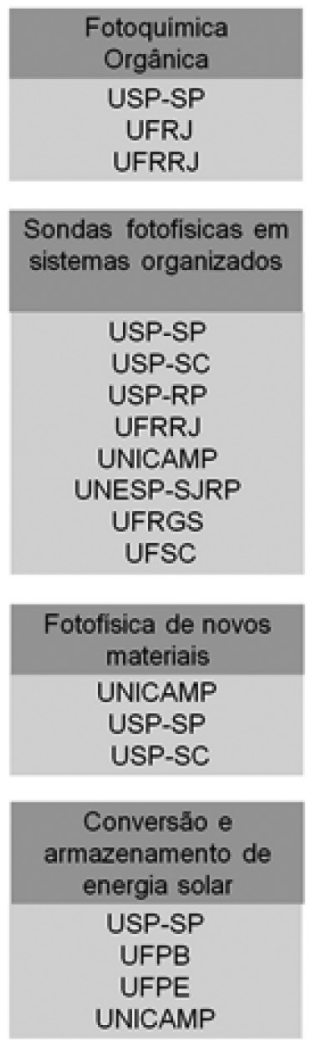
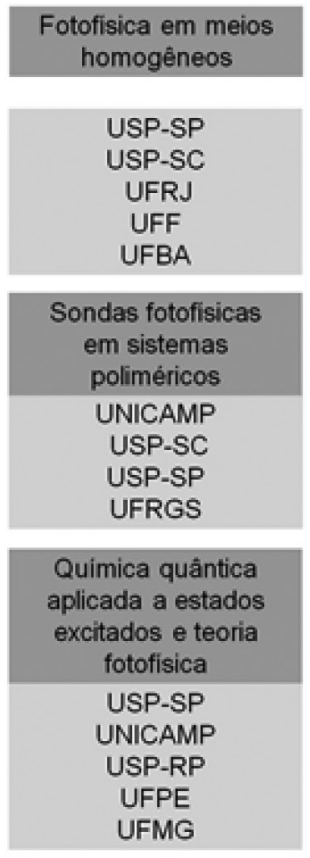

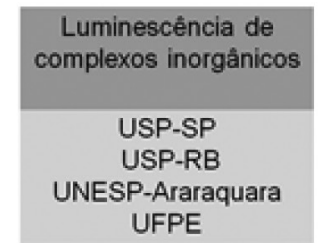

Sondas fotofisicas

associadas com

macromoléculas

biológicas

UFRJ

USP-SC

UNIFESP

USP-SP

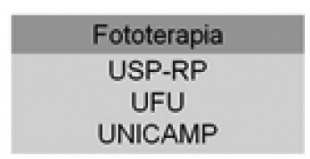

Fotoquimica ambiental

UNICAMP

UFRJ

USP-SP

UFBA
Fotoquimica de

polimeros e processos

e fotopolimerizaçăo

USP-SP

USP-SC
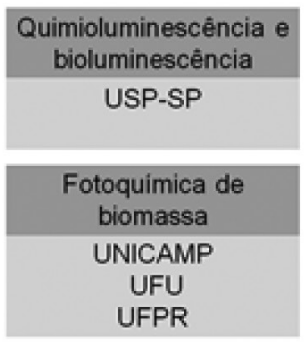

Figura 4. Principais áreas de estudo desenvolvidas no final da década de 90 no Brasil ${ }^{1}$ 
mecanismo fotoquímicos e fotofísicos não seriam mais alvos centrais na pesquisa e que o foco seria o interesse no uso das técnicas fotofísicas em estudos básicos de dinâmica, mobilidade, microestrutura e reatividade em sistemas organizados e macromoleculares com a finalidade de produzir intermediários reativos. Uma preocupação dos pesquisadores na época era de que a interdisciplinaridade e aplicações de metodologia da fotoquímica em outras áreas do conhecimento acabaria criando uma certa crise de identidade para os fotoquímicos.

$\mathrm{Na}$ época já havia uma forte tendência de fotoquímicos que se identificavam com áreas que utilizavam a aplicação de metodologia fotofísica à solução de seus problemas (p. ex., coloides e materiais). Além disso, os pesquisadores destas áreas passaram a utilizar a metodologia fotofísica como um instrumento, mas não pertenciam à comunidade fotoquímica. A questão chave da época era saber se a fotoquímica teria um mecanismo de sobrevivência como um campo específico do conhecimento.

\section{AVANÇOS NOS ÚLTIMOS 15 ANOS}

Nestes últimos 15 anos a área de fotoquímica do país tem demonstrado que continua competitiva no cenário internacional e que encontrou os mecanismos necessários para permanecer como uma área especifica em meio a um oceano interdisciplinar. Um levantamento utilizando como base o banco universal de dados do ISI, através da Web-of-Science no período de 2000 até 2016 empregando-se o termo photochem* e que incluíam o Brazil no endereço de um dos autores apresenta a evolução no número de publicações na área realizadas por pesquisadores brasileiros. Embora números apresentados possam ser algo maiores (devido à possível omissão de alguns trabalhos), podem servir para se ter uma ideia global do crescimento. A Figura 5 apresenta o número de publicações por ano relacionados às duas palavras-chaves de busca. Observa-se que por volta do ano 2000 havia 30 publicações por ano. Com o decorrer dos anos houve um crescimento bastante acentuado chegando em 2016 a 127 publicações/ ano. Deve-se ressaltar que, neste levantamento, não foram levados em conta trabalhos que tratam apenas da aplicação de técnicas rotineiras

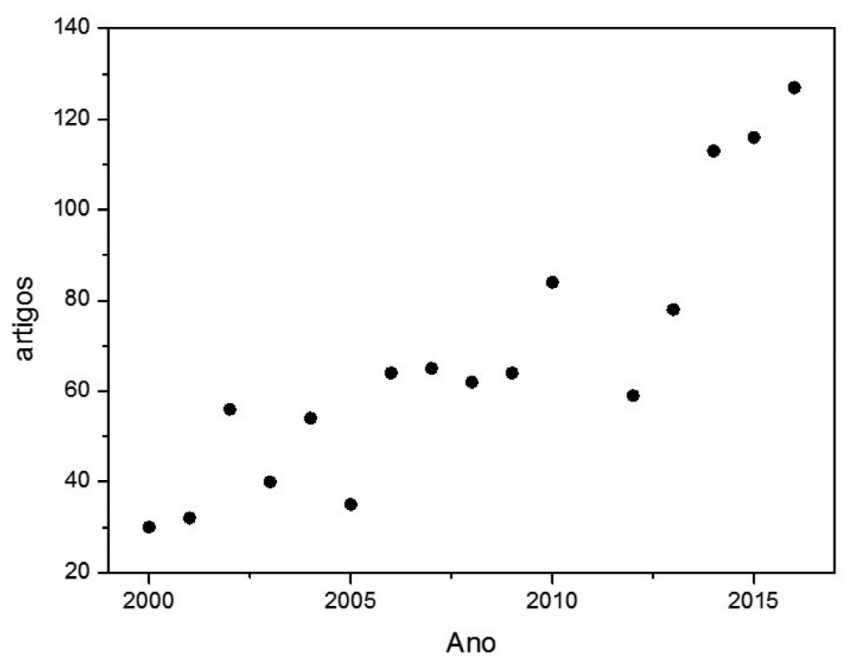

Figura 5. Crescimento do número de publicações nas diversas especialidades de fotoquímica no Brasil em revistas internacionais. Os dados foram extraídos do banco universal de dados do ISI, através da Web-of-Science no período de 2000 até 2016 empregando-se o termo photochem* incluindo Brazil no endereço de um dos autores

com aplicação analíticas e bioquímicas, que em geral não mencionam no título, ou nas palavras chaves, os termos photochem*.

Em relação às atuais linhas de pesquisa, no país, é possível constatar por meio de um levantamento na Plataforma Lattes que, apesar de existir uma força na direção de tornar a fotoquímica um instrumento para resolver questões de diversas áreas da ciência como havia sido previsto anteriormente, algumas das áreas ainda continuam sendo o objeto de investigação para diferentes grupos de pesquisa conforme pode ser observado na Tabela 2. Os grupos mais tradicionais de São Paulo e Rio de Janeiro continuam a desenvolver pesquisas básicas, ao mesmo tempo que estenderam suas linhas de pesquisa a assuntos de ciência aplicada. Além disso, novos grupos surgiram nas regiões centro-oeste, sul e nordeste.

Tabela 2. Áreas dominantes envolvendo fotoquímica no Brasil atualmente

\begin{tabular}{|c|c|}
\hline Área & Centro \\
\hline Processos Oxidativos Avançados & $\begin{array}{l}\text { USP-SP, USP-São Carlos, UNICAMP, UERJ, UFRGS, UFMS, UFPe, UFRN, UNESP, UNESP-Araraquara, } \\
\text { UFRGS, UFRG }\end{array}$ \\
\hline Fotobioquímica e Fotobiologia & USP-SP, USP-Ribeirão Preto \\
\hline Fototerapia dinâmica & USP-SP, USP-Ribeirão Preto, UFABC, UNIVAP, UEM, UnB \\
\hline Fotoquímica inorgânica & USP-São Paulo, USP-Ribeirão Preto, UFSCar, UFU, UFC \\
\hline Fotoquímica orgânica & USP-São Paulo, USP-São Carlos, UFRRJ, UFRJ, UFRGS, UFMS \\
\hline Fotodegradação & $\begin{array}{l}\text { USP-São Paulo, USP-São Carlos, UNESP- Araraquara, UNICAMP, UENFlum, UFMG, UFTriangMineiro, } \\
\text { UFU, UFPR, UNIOESTE-Toledo, UFRGS, UFMS, UFCG, UFRG, UFOB }\end{array}$ \\
\hline Fotoconversão de energia/células solares & USP-São Paulo, UNICAMP, UFRGS \\
\hline Fotobiofísica & USP-Ribeirão Preto \\
\hline Fotopolimerização & USP-São Carlos, UNESP- Pres. Prudente \\
\hline Fotoquímica de alimentos & USP-São Carlos \\
\hline Fotocatálise & USP-São Carlos, UFU, UNIOESTE-Toledo, UFRGS, UFPE, UFOB, UFBA \\
\hline Fotossíntese & $\begin{array}{l}\text { UNICAMP, Instituto Agronômico de Campinas, UNIVAP, UFRRJ, UENFlum, UFES, UFSC, UFPEL, UFC, } \\
\text { UVV-ES }\end{array}$ \\
\hline Espectroscopia de moléculas individuais & UNICAMP, USP-São Carlos \\
\hline Fotoeletroquímica & UFTriangMineiro, UFRGS, \\
\hline Fotofísica em sistemas micro-heterogêneos & USP-São Paulo, USP-São Carlos, UFSC \\
\hline
\end{tabular}


Assim, esses grupos vêm consolidando atividades de pesquisa básica em fotoquímica e/ou investigando interações com diversas áreas interdisciplinares mostrando a relevância da aplicação das técnicas de fotoquímica. Nesse sentido, podem se observar estudos básicos de fotofísica e fotoquímica de moléculas orgânicas, que incluem o desenvolvimento de estudos que levam à compreensão da relação entre a química e a fotoquímica e a função biológica de corantes e pigmentos naturais; propostas de mecanismos gerais que permitam entender os aspectos básicos das reações de degradação fotocatalisada de poluentes orgânicos; e as atividades antioxidantes e seu papel em fotoprocessos. De outro lado, o exame de aspectos fundamentais e aplicados de reações de fotopolimerização e fotoquímica de polímeros foram estudados em vários aspectos: a cinética e fotoiniciação de polimerização é um de grande importância em muitas aplicações práticas, tais como, resinas dentárias e tintas. Entretanto, como existiam poucos estudos sobre os parâmetros fundamentais das reações elementares envolvidas nesses processos, foram realizados estudos para determinar esses parâmetros e entender os mecanismos globais de polimerização e estabelecer correlações entre as condições das polimerizações fotoiniciadas e as propriedades dos materiais resultantes. O mesmo se aplica às reações envolvidas na fotodegradação de polímeros. Outro campo de crescente interesse é a Terapia Fotodinâmica. Existem muitos estudos clínicos e pré-clínicos relacionados ao uso da terapia fotodinâmica para o tratamento de câncer de pele, modulação da resposta dos processos de cicatrização cutânea e indução da restauração das terminações nervosas e crescimento neurônico em órgãos periféricos. Outro objeto de investigação em relação a este assunto é o desenvolvimento de sistemas nanoestruturados, ou baseados em nanopartículas, para targeting de agentes terapêuticos, com foco nos alvos de interesse biológico e avaliação da fotoquímica e fotofísica destes sistemas. Avaliação das eficiências in vivo e in vitro. Estudos mecanísticos de sistemas quimioluminescentes para compreender os princípios gerais que controlam a eficiência (rendimento quântico) das transformações, que poderiam permitir o desenvolvimento de sistemas mais eficientes e o planejamento racional das aplicações destes sistemas. Estudos do mecanismo da bioluminescência de fungos e o aperfeiçoamento de testes ecotoxicológicos para a determinação da toxidez das substâncias químicas com relação a estes fungos.

Em relação à instrumentação destaca-se que existem equipamentos no país que tornam possíveis pesquisas na área de fotoquímica com competitividade internacional. As técnicas atualmente disponíveis na comunidade fotoquímica no país são: Fluorescência de picossegundo resolvida no tempo, microscopia de fluorescência de dois-fótons, fluorescência de nanossegundo resolvida no tempo e fotólise por pulso de laser em nanossegundos, espectroscopia de femtossegundos e emissão de oxigênio singlete resolvida no tempo e no espaço, espectroscopia de ressonância de plásmon e fotocatálise induzida por ressonância de plásmon e espectroscopia de massa acoplada a espectroscopia de laser.

Também vale a pena mencionar o alto nível de inserção dos pesquisadores da área de fotoquímica no panorama internacional. Devido às frequentes e intensas interações de nossos fotoquímicos com grupos do exterior, é habitual existirem projetos em cooperações internacionais, tanto com outros países de América Latina, como com Estados Unidos, Canadá e Europa. Adicionalmente, membros da nossa Comunidade têm ocupado cargos em Organismos Internacionais relacionados com a Fotoquímica, como a Inter-American Photochemical Society (I-APS) e a European Photochemical Association (EPA), tendo sido responsáveis pela organização de Congressos. A isto pode também agregar-se a atuação de quase todos os Professores envolvidos na área como Editores, Assessores científicos de publicações e avaliadores de projetos internacionais.

\section{REFERÊNCIAS}

1. Neumann, M. G.; Quina, F. H.; Quim. Nova 2002, 25, 34.

2. Riveros, J. M.; Em Paschoal Senise, Uma Carreira Dedicada à Educação; Colli, W., ed.; Paralelo 15 Ed. e CAPES, MEC: Brasília, 2001, p. 73.

3. Braslavsky, S. E.; Simon, J. D.; Photochem. Photobiol 2005, 81,768 